\title{
Cyclotron resonant scattering feature simulations
}

\section{Thermally averaged cyclotron scattering cross sections, mean free photon-path tables, and electron momentum sampling ${ }^{\star}$}

\author{
F.-W. Schwarm ${ }^{1}$, G. Schönherr ${ }^{2}$, S. Falkner ${ }^{1}$, K. Pottschmidt ${ }^{3}, 4$, M. T. Wolff ${ }^{5}$, P. A. Becker ${ }^{6}$, E. Sokolova-Lapa ${ }^{7,8}$, \\ D. Klochkov ${ }^{9}$, C. Ferrigno ${ }^{10}$, F. Fürst ${ }^{11}$, P. B. Hemphill ${ }^{12}$, D. M. Marcu-Cheatham ${ }^{3,4}$, T. Dauser ${ }^{1}$, and J. Wilms ${ }^{1}$ \\ 1 Dr. Karl Remeis-Sternwarte and Erlangen Centre for Astroparticle Physics, Sternwartstrasse 7, 96049 Bamberg, Germany \\ e-mail: fritz.schwarm@sternwarte.uni-erlangen.de \\ 2 Leibniz-Institut für Astrophysik Potsdam (AIP), An der Sternwarte 16, 14482 Potsdam, Germany \\ 3 CRESST, Department of Physics, and Center for Space Science and Technology, UMBC, Baltimore, MD 21250, USA \\ 4 NASA Goddard Space Flight Center, Code 661, Greenbelt, MD 20771, USA \\ 5 Space Science Division, Naval Research Laboratory, Washington, DC 20375-5352, USA \\ 6 Department of Physics \& Astronomy, George Mason University, Fairfax, VA 22030-4444, USA \\ 7 Faculty of Physics, M. V. Lomonosov Moscow State University, Leninskie Gory, 119991 Moscow, Russia \\ 8 Sternberg Astronomical Institute, Moscow M. V. Lomonosov State University, Universitetskij pr., 13, 119992 Moscow, Russia \\ 9 Institut für Astronomie und Astrophysik, Universität Tübingen (IAAT), Sand 1, 72076 Tübingen, Germany \\ 10 ISDC Data Center for Astrophysics, Université de Genève, chemin d'Écogia 16, 1290 Versoix, Switzerland \\ 11 Cahill Center for Astronomy and Astrophysics, California Institute of Technology, Pasadena, CA 91125, USA \\ 12 Center for Astrophysics and Space Sciences, University of California, San Diego, 9500 Gilman Dr., La Jolla, \\ CA 92093-0424, USA
}

Received 20 July 2016 / Accepted 30 August 2016

\begin{abstract}
Context. Electron cyclotron resonant scattering features (CRSFs) are observed as absorption-like lines in the spectra of X-ray pulsars. A significant fraction of the computing time for Monte Carlo simulations of these quantum mechanical features is spent on the calculation of the mean free path for each individual photon before scattering, since it involves a complex numerical integration over the scattering cross section and the (thermal) velocity distribution of the scattering electrons.

Aims. We aim to numerically calculate interpolation tables which can be used in CRSF simulations to sample the mean free path of the scattering photon and the momentum of the scattering electron. The tables also contain all the information required for sampling the scattering electron's final spin.

Methods. The tables were calculated using an adaptive Simpson integration scheme. The energy and angle grids were refined until a prescribed accuracy is reached. The tables are used by our simulation code to produce artificial CRSF spectra. The electron momenta sampled during these simulations were analyzed and justified using theoretically determined boundaries.

Results. We present a complete set of tables suited for mean free path calculations of Monte Carlo simulations of the cyclotron scattering process for conditions expected in typical X-ray pulsar accretion columns $\left(0.01 \leq B / B_{\text {crit }} \leq 0.12\right.$, where $B_{\text {crit }}=4.413 \times$ $10^{13} \mathrm{G}$, and $3 \mathrm{keV} \leq k_{\mathrm{B}} T \leq 15 \mathrm{keV}$ ). The sampling of the tables is chosen such that the results have an estimated relative error of at most $1 / 15$ for all points in the grid. The tables are available online (see link in footnote, page 1).
\end{abstract}

Key words. X-rays: binaries - stars: neutron - methods: numerical

\section{Introduction}

Cyclotron resonant scattering features (CRSFs, often also called "cyclotron lines") have been measured in numerous accreting $\mathrm{X}$-ray pulsars and are the only direct way to measure a neutron star's magnetic field. They result from the interaction of photons with electrons in the presence of strong $B$-fields approaching the critical field strength,

$B_{\text {crit }}=\frac{m_{\mathrm{e}}^{2} c^{3}}{e \hbar}=4.413 \times 10^{13} \mathrm{G}$

\footnotetext{
* The electronic tables described here are available at http://www sternwarte.uni-erlangen.de/research/cyclo
}

where $m_{\mathrm{e}}$ is the electron rest mass, $e$ its charge, and $c$ the speed of light. Their positions can be estimated as $\sim n E_{\text {cyc }}(n=1,2,3, \ldots)$ using the $12-B-12$ rule, $E_{\text {cyc }} \approx 12 B_{12} \mathrm{keV}$, with the $B$-field strength $B_{12}$ given in units of $10^{12} \mathrm{G}$. Cyclotron lines have their origin in transitions of electrons between different Landau levels, which are the discrete energy states an electron can occupy within such a strong magnetic field.

The electrons are quantized perpendicular to the field and therefore give rise to quantum mechanical absorption and resonant scattering processes altering the spectral and spatial distribution of the participating photons. The probability for an interaction to occur is given by the corresponding cyclotron cross section. In the course of simulating this process with Monte Carlo (MC) methods, these cross sections can be used 
to sample the mean free path of a photon within such a medium. We have separated this core issue from the larger simulation code to allow for an efficient simulation of any complex X-ray pulsar geometry based on precalculated tables of the mean free path.

In the following we describe the calculation method and usage of these mean free path interpolation tables and discuss how the sampling of electron parallel momenta influences the formation of CRSFs. In Sect. 2 we discuss the necessity of mean free path interpolation tables and their usage and introduce their computation and the interpolation mechanism. In Sect. 3 we explain the importance of the sampling of the electron parallel momentum. In particular we illustrate the connection between cyclotron resonances and the corresponding behavior of the sampled electron parallel momenta, since the understanding of these parameters is essential for the application of the Monte Carlo code to generate synthetic spectra. Many more applications can be envisioned, including the simulation of the influence of cyclotron scattering on the electrons, or the overall accretion geometry. Here, we restrict ourselves to the discussion of the mean free path interpolation tables. Their motivation and application is described against the background of Monte Carlo simulation of cyclotron lines. The description and application of the full MC scattering code, which has been written with the prime goal of imprinting cyclotron lines on the continuum emission of astronomical X-ray sources, and which includes a working fit model, will be the subject of a forthcoming publication (Schwarm et al., in prep., hereafter Paper II). Compared to previous MC simulations, it allows for much more complex physical scenarios, the exploration of which is the goal of this series of papers.

\section{Mean free path interpolation tables}

\subsection{Motivation}

When simulating synthetic cyclotron line spectra using an MC method, a photon with an initial energy is generated. Then the optical depth $\tau$ to be traveled by the photon is drawn from the exponential distribution $\exp (-\tau / \lambda)$, and converted into a geometric path length. This requires the calculation of the mean free path $\lambda$, that is, the inverse thermally averaged scattering cross section. The photon is then propagated over this distance and the scattering process is simulated. This simulation requires us to choose an electron that has properties appropriate for this mean free path (MFP). Paper II and Schwarm et al. (2012) show a flow chart of the full MC scattering process. Since the calculation of the geometric path and the scattering simulation are very time consuming, we have developed a tabular interpolation scheme for the mean free path and electron parallel momentum sampling to save computing time. It works on precalculated tables, which were obtained using an adaptive process refining the table until the interpolation error is smaller than a preset limit.

The mean free path of a photon in a CRSF medium is the inverse of the sum over the cross sections of all possible CRSF related interactions between the photon and its possible scattering partners, which throughout this work are assumed to be only electrons (see Eq. (4) below). This calculation not only involves a summation over all possible final Landau levels and spin states of the electrons, but also a summation over all possible intermediate states. Furthermore, the electrons have a temperature dependent continuous momentum distribution parallel to the magnetic field, which leads to Lorentz boosting of the scattering photons in the electrons' rest frames giving rise to an integration over possible initial electron parallel momenta as well. Finally, the cross sections are summed over final polarization states and averaged over the initial ones for a polarization averaged mean free path. Photons are either in ordinary or extra-ordinary polarization mode. The modes differ in the orientation of a photon's electric field vector with respect to the plane defined by the direction of motion and the external magnetic field (Canuto et al. 1971; Mészáros \& Ventura 1978; Becker \& Wolff 2007).

Calculating the mean free path is only the first step in the MC sampling process, but replacing it by an interpolation scheme has a very large impact on the overall simulation time. The CRSFs can form at rather small optical depths due to the resonant nature of the cross sections. The small optical depths as considered here mean that most of the injected seed photons escape the CRSF medium immediately. Only $1-10 \%$ of the initial photons will interact with the medium via cyclotron scattering. Therefore the only evaluation necessary for the majority of photons is the one for their initial mean free path.

The simulations show that interacting photons tend to scatter around the resonances in energy space until an electron with parallel momentum that deviates sufficiently from the resonance condition is hit. Spawned photons, that is, photons emitted by previously excited electrons during their transition to a lower Landau level, are also generated close to the resonances. This leads to a large number of resonant photons compared to continuum photons and further motivates the usage of the interpolation tables presented here since the calculation time tends to increase significantly near the resonances.

For each interaction between photons and electrons, the parallel momentum of the electron must be sampled according to the corresponding thermal momentum distribution. For a given photon energy and angle, the electrons with momenta that cause the photon to be resonant in the electron rest frame have a much higher scattering probability. Therefore the cyclotron resonances manifest in parallel momentum resonances of the scattering electrons (see Sect. 3).

The mean free path tables presented here store the total mean free paths as well as the probability distributions needed for sampling the electron momentum. Interpolating from these tables speeds up our simulation by a factor of $\sim 60$ compared to the case where all data needed during the simulation is calculated without resorting to interpolation tables. The difference between interpolation and calculation becomes even larger, if the tables are used outside of the simulation to simply interpolate mean free paths for several energies without performing any other simulation steps. In this case interpolation is faster by a factor of $\sim 2400$ for input angles almost perpendicular to the magnetic field.

\subsection{Calculation of the averaged cross sections}

The calculation of the mean free path needed for the sampling of propagation lengths of photons within a scattering medium relies on the integration of the scattering cross section $\sigma$ over a range of electron momenta $p$, effectively averaging the cross section over the parallel electron temperature (Daugherty \& Harding 1986),

$\langle\sigma(\omega, \mu)\rangle_{f_{\mathrm{e}}}=\int_{-\infty}^{+\infty} \mathrm{d} p f_{\mathrm{e}}(p, T)(1-\mu \beta) \sigma_{\mathrm{rf}}\left(\omega_{\mathrm{rf}}, \mu_{\mathrm{rf}}\right)$,

where $\mu=\cos \vartheta, \vartheta$ is the angle between the photon's direction and the magnetic field, $\omega$ is the photon energy, and $\beta=v / c$. The subscript "rf" refers to values in the rest frame of the electron. $f_{e}(p, T)$ is the electron's momentum distribution, chosen here as a relativistic Maxwellian with temperature $T$ :

$f_{\mathrm{e}}(p, T) \propto \exp \left(-\frac{1}{k_{\mathrm{B}} T}\left(\sqrt{m_{\mathrm{e}}^{2} c^{4}+p^{2} c^{2}}-m_{\mathrm{e}} c^{2}\right)\right)$. 
The mean free path, $\lambda$, is then given by (Araya \& Harding 1999)

$$
\lambda(\omega, \mu)=1 /\langle\sigma(\omega, \mu)\rangle_{f_{\mathrm{e}}} .
$$

The fully relativistic differential Compton scattering cross section, $\sigma_{\mathrm{rf}}$, used here was derived by Bussard et al. (1986) and Sina (1996). These authors used the Breit-Wigner broadening approximation and electron wave functions from Sokolov et al. (1968), which are consistent with the perturbation theoretic order of the calculation and the expected "time dilation" relation (Graziani 1993). Daugherty \& Ventura (1978) presented an alternative derivation of the cross sections using electron wave functions from Johnson \& Lippmann (1949). See Herold et al. (1982) for the relativistic transition rates, which are needed for the cross section calculation and for the sampling of the final Landau level into which an initially excited electron decays.

We use a revised implementation of the code developed by Sina (1996) for the calculation of the scattering cross sections. His original code is used in the MC simulation from Araya (1997), which has been employed by Schönherr et al. (2007) to calculate the Green's functions necessary for their CRSF fitting model. The thermally averaged cross sections used in the Araya (1997) code differ from the ones calculated by Harding \& Daugherty (1991; Schwarm et al. 2012). We verified that the origin of this difference lays in the integration of the cross section code into the MC simulation rather than the cross section calculation itself, by succeeding to reproduce the Harding \& Daugherty (1991) profiles with our revised implementation of the Sina (1996) code. The code has been restructured to minimize code repetition and increase readability. It has been generalized to allow for previously hard-coded variables, such as numerical integration boundaries and methods, or the maximum number of Landau levels taken into account, to be changed dynamically. It has been extended to arbitrary temperatures by including an adaptive Simpson integration scheme for averaging the cross sections over the thermal electron momentum distribution. Improved error handling and the addition of warning messages result in a more robust implementation as needed for the time consuming MFP table calculations. Unit tests ensure that the resulting cross sections are in agreement with the ones calculated by the original code. The resulting cross sections are also in agreement with the work of Gonthier et al. (2014). These authors derived the cross section for the special, $\vartheta=0$, case of photons propagating parallel to the magnetic field, following the approach of Sina (1996) as well.

The cross sections are sharply peaked functions, which are difficult to integrate numerically. Figure 1 shows an example for transitions from the Landau ground state with initial spin $-1 / 2$ to a final state with negative spin summed over the first five possible final Landau levels. A slight shift of the position of the resonances to higher energies occurs for smaller angles of the photon to the magnetic field.

The numerical evaluation of Eq. (2) is closely related to the evaluation of the probability distribution of the electron momentum (Araya \& Harding 1999),

$F(p) \propto \int_{-\infty}^{p} \mathrm{~d} p^{\prime} f_{\mathrm{e}}\left(p^{\prime}, T\right)(1-\mu \beta) \sigma_{\mathrm{rf}}\left(\omega_{\mathrm{rf}}, \mu_{\mathrm{rf}}\right)$,

which is required in Monte Carlo simulations in order to find the momentum of the photon's scattering partner. The total inverse mean free path, calculated by Eq. (2), accounts for all possible electron momenta and is used to normalize $F(p)$ to unity. By searching for the momentum $p$ for which $F(p)=\mathrm{Rn}$, with a random number $0 \leq \mathrm{Rn} \leq 1$, the parallel momentum of the scattering electron can be sampled.

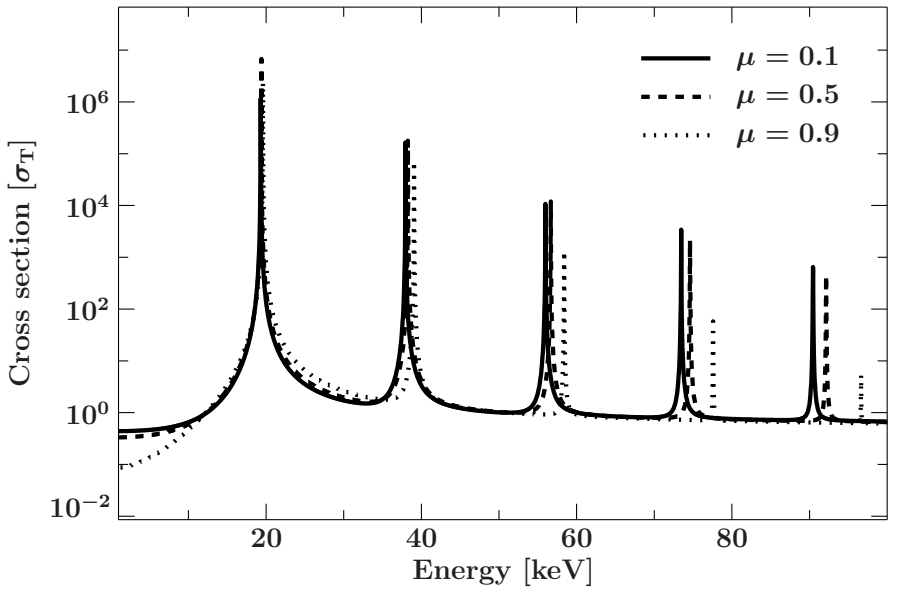

Fig. 1. Scattering cross section as a function of rest frame energy for exciting an electron in initial Landau level $n_{\mathrm{i}}=0$ with spin $-1 / 2$ to level $n_{\mathrm{f}}$ with $n_{\mathrm{i}} \leq n_{\mathrm{f}} \leq 6$ and final spin $-1 / 2$ within a magnetic field of strength $B=0.0385 B_{\text {crit }}$, resulting in a fundamental cyclotron resonance at $\sim 20 \mathrm{keV}$. The solid, dashed, and dotted lines show cross sections for $\mu=\cos \vartheta=0.1,0.5,0.9$, corresponding to angles between the path of the incoming photon and the $B$-field of $84.2,60^{\circ}$, and 26.8 , respectively.

In order to calculate $F(p)$ for a given magnetic field, temperature, photon energy, and angle, we use an adaptive Simpson integrator (McKeeman 1962). The thermal momentum distribution $f_{\mathrm{e}}(p, T)$ becomes very small for large absolute values of the electron momentum. Therefore the numerical integration limits were set to $\sim \pm m_{\mathrm{e}} c^{2}$. They cover a range much larger than the expected momentum range of the electrons. This way the $45 k_{\mathrm{B}} T$ boundaries used by, for example, Araya \& Harding (1999) are also covered for the temperature range of $3 \mathrm{keV}$ to $15 \mathrm{keV}$. In our adaptive approach the integration interval is successively split into smaller intervals. The integrals of these intervals are approximated by integrating a suitable third order polynomial. Due to the adaptive nature of the integration method, the larger momentum range we use compared to earlier works leads to only marginally increased computing time.

The splitting of the integration intervals has to be stopped when a suitable convergence criterion is fulfilled. Lyness (1969) shows that in fifth order approximation the maximum error of the integrator can be estimated as

$\epsilon=\frac{1}{15} \sum_{i} F_{a_{i}}^{c_{i}}-\left(F_{a_{i}}^{b_{i}}+F_{b_{i}}^{c_{i}}\right)$,

where $F_{a_{i}}^{c_{i}}$ and $\left(F_{a_{i}}^{b_{i}}+F_{b_{i}}^{c_{i}}\right)$ are the numerical estimates for the integrals over the interval $\left[a_{i}, c_{i}\right]$ before and after bisecting the interval. We choose a relative maximum error of $1 / 15$ as a convenient compromise between accuracy, calculation time, and table size. The error is halved in each recursion step to ensure that the total error estimate remains below the chosen maximum error for the total interval.

\subsection{Optimizing table size: adaptive calculation}

The adaptive nature of this integration method takes advantage of the fact that only few integration points are needed outside the resonances. These choices allow for a computationally manageable creation of mean free path tables on a time scale of 3000-30000 CPU hours on a typical work station with processor speed of $2-3 \mathrm{GHz}$, depending on the magnetic field, 


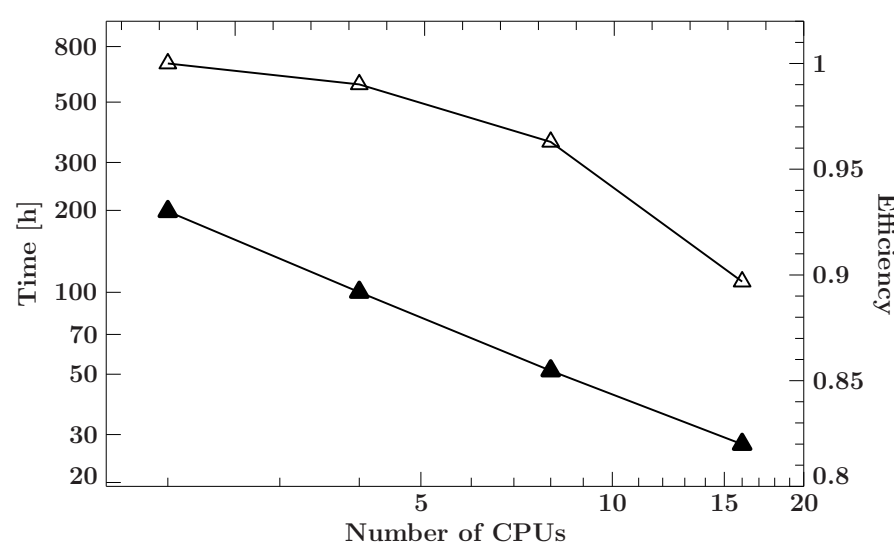

Fig. 2. Absolute CPU time (filled symbols) on the left axis and multicore efficiency (open symbols) on the right axis for MFP table calculation. The efficiency is calculated as $\eta=t_{1} /\left(n t_{n}\right)$, where $t_{n}$ is the execution time on $n$ CPUs. $t_{1}=2 t_{2}$ is assumed since performing the calculation on only one core is too time consuming. The MFP table calculations were performed on an AMD Opteron $2.2 \mathrm{GHz}$ system. The execution times are for a magnetic field $B=0.12 B_{\text {crit }}$ and a temperature $k_{\mathrm{B}} T=3 \mathrm{keV}$ with a maximum error of $2 / 15$. The CPU time scales with the table file size (see Sect. 2.4).

the temperature, and the error tolerance. These calculation times are achieved by utilizing a few dozen CPU cores ${ }^{1}$ at the same time using the Message Passing Interface (MPI; MPI Forum 1994). Parallelization leads to a significant speed up by a factor of up to $0.8 N_{\mathrm{CPU}}$ with respect to earlier implementations, where $N_{\text {CPU }}$ is the number of CPUs used. Figure 2 shows this speed up as a function of $N_{\mathrm{CPU}}$ in terms of the parallel computing efficiency, that is, the total CPU time used for a mean free path table calculation or for a simulation divided by the CPU time needed on only one CPU.

As discussed in Appendix A, for the fast interpolation of the mean free path we evaluate the integrals using another adaptive process where the energy and the angular grid points are iteratively refined until the mean free paths interpolated from the table do not deviate from the corresponding calculated ones by more than a given maximum deviation. After each calculation step of a new energy grid point, the result is compared to the value obtained by linear interpolation. The relative error of the interpolation is determined and used as a convergence criterion. A minimum recursion depth as well as maximal angle and energy differences prevent premature convergence.

The partial integrals, forming the cumulative distribution function (CDF) of the electron momentum, are stored in FITS binary tables allowing for efficient loading into a simulation. FITS tables can be opened and read by many processes in parallel, enabling the usage of parallel computation in simulations. Another advantage of the FITS format is the efficient caching provided by modern FITS libraries such as cfitsio ${ }^{2}$, which minimize the bottleneck of disk reading operations. Although the whole size of such an interpolation table is on the order of 1-200 GB for the currently required accuracy, and thus will not completely fit into memory for most common computers, for a given setup the resonant nature of the scattering process means that the most frequently required mean free paths will be from a small fraction of the whole table only, which fits completely into memory and therefore reduces the number of disk reads dramatically.

\footnotetext{
1 For the sake of simplicity the terms "CPU" and "CPU core" are used equivalently throughout this work.

2 http://heasarc.nasa.gov/fitsio/fitsio.html
}

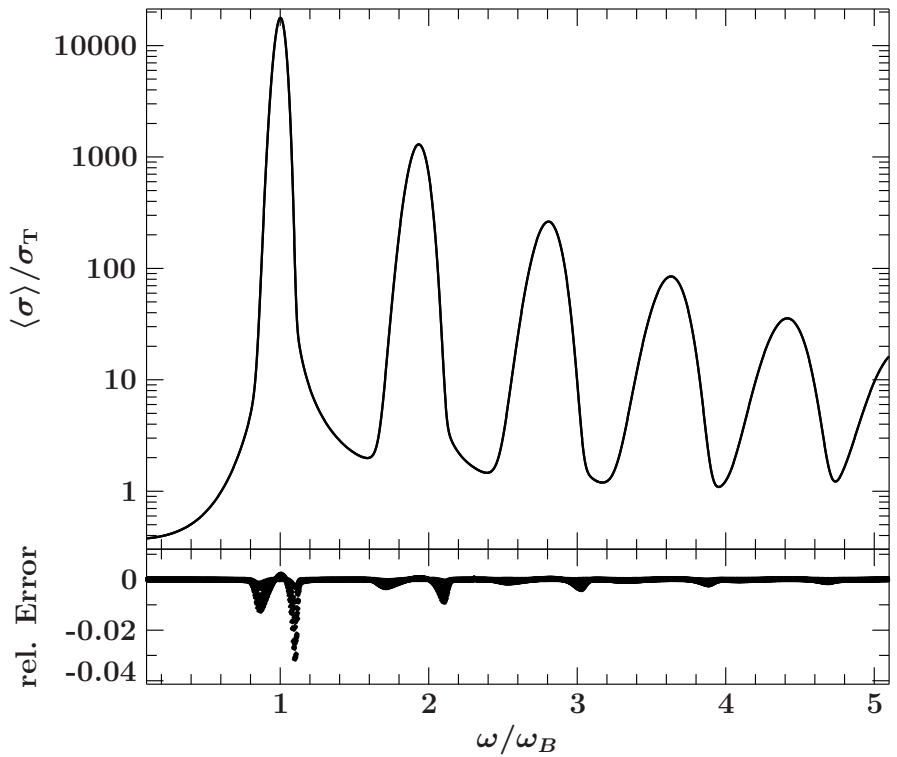

Fig. 3. Thermally averaged cyclotron scattering cross sections in units of the Thomson cross section $\sigma_{\mathrm{T}}$ interpolated for $B=0.12 B_{\text {crit }}, k_{\mathrm{B}} T=$ $3 \mathrm{keV}$, and angle $\vartheta=60^{\circ}$. The relative deviations to the calculated profiles, shown in the lower panel, are not exceeding the maximum error of $1 / 15$ of the MFP table used for the interpolation.

For testing the accuracy of the interpolation from the resulting tables, we calculated the thermally averaged scattering cross section profiles (Eq. (2)) on a much finer grid than the tabulated one ( 700-1800 energy grid points). Figure 3 visualizes the profile for a magnetic field of $B=0.12 B_{\text {crit }}$, interpolated on a grid of 5000 energy grid points. The relative deviations from the calculated values do not exceed the relative maximum error of $1 / 15$.

Appendix B shows interpolated profiles for the parameter combinations for which tables are provided. They are plotted as a function of frequency and scattering angle for several $B$-fields and temperatures in Figs. B.1 and B.2.

\subsection{Parameter ranges and file sizes}

The electronic data provided with this publication is available online (see link in footnote, page 1). We provide tables for the mean free path calculated for $B$-fields and temperatures covering the parameter ranges typical for accreting X-ray pulsars, namely $B=0.01,0.03,0.06,0.09$, and $0.12 B_{\text {crit }}$ and $k_{\mathrm{B}} T=3,6,9$, 12 , and $15 \mathrm{keV}$. Table B.2 shows a list of the available tables and their uncompressed file size. As shown by Araya \& Harding (1999), the low continuum optical depth and the small collisional excitation rate (Bonazzola et al. 1979; Langer 1981) of a typical accretion column, combined with the high radiative cyclotron de-excitation rate (Latal 1986), imply that we can assume that all electrons are initially in the ground state Landau level and have a relativistic thermal momentum distribution, which is not altered by cyclotron resonant scattering. We therefore provide tables for this case only. The full data set has an uncompressed size of approximately 2.7 Terabytes. Compressing the tables using gzip leads to a size reduced by roughly $50 \%$.

The number of momentum grid points needed for convergence of the numerical integration of Eq. (2), correlates positively with the resulting inverse mean free path. Thus broader profiles and those with larger absolute values require more $p$-grid points. The number of energy grid points needed for accurate interpolation strongly depends on the complexity of the 
F.-W. Schwarm et al.: Cyclotron resonant scattering feature simulations. I.

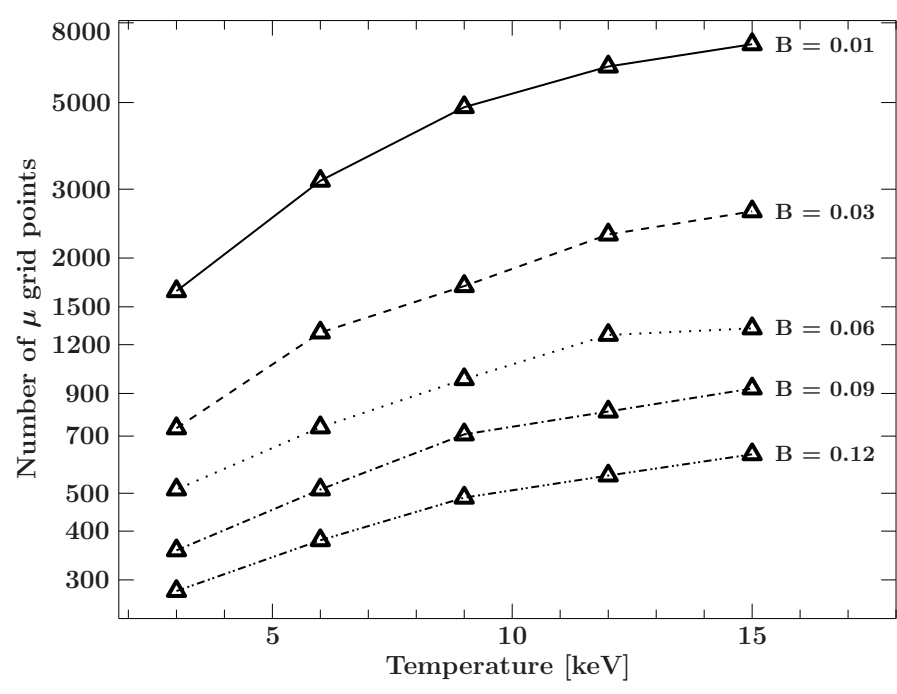

Fig. 4. Number of $\mu$-grid points with respect to the electron temperature. Each line corresponds to one magnetic field strength.

profile and therefore on the scattering angle. The sharply peaked profiles for angles $\sim 90^{\circ}$ to the magnetic field require many more angle grid points. The average number of energy grid points for each table does not depend on the temperature, nor does it vary with the magnetic field strength for all but the highest field, $B=0.12 B_{\text {crit }}$. For this field the tables have twice as many energy grid points as the other tables. The number of angle grid points in each table is correlated with the temperature and the magnetic field strength. More $\mu$-grid points are needed for higher temperatures since the broadening of the profiles towards larger $\mu$ increases with temperature. The negative correlation with magnetic field strength can be explained by the larger absolute values of the corresponding profiles. Figure 4 shows the number of $\mu$-grid points, in the set of MFP tables presented here, as a function of the electron temperature and the magnetic field strength. The largest table was obtained for the lowest magnetic field strength and the highest temperature, and is $238 \mathrm{~GB}$ in size.

\section{The sampling of the electron parallel momentum}

The electrons' momenta perpendicular to the magnetic field are quantized. The momenta parallel to the $B$-field are distributed according to Eq. (3). The integration of the product of this distribution with the corresponding scattering cross sections leads to the cumulative distribution function (Eq. (5)). During the MC simulation the scattering electron's parallel momentum is drawn from this distribution. The influence of this sampling process on the cyclotron line shape is illustrated in Fig. 5. It shows the momenta drawn during a simulation with respect to the scattering photons' energies. The probability for photons to scatter off an electron with the right momentum to make the photon fulfill the resonance condition in the electron's rest frame is much higher. Therefore the events shown in Fig. 5 track the resonance condition (see, e.g., Daugherty \& Ventura 1978; Harding \& Daugherty 1991)

$$
\sqrt{p^{2} c^{2}+m_{\mathrm{e}}^{2} c^{4}}=p c \cos \vartheta+n \frac{B}{B_{\text {crit }}} \frac{m_{\mathrm{e}}^{2} c^{4}}{\omega}-\frac{\omega}{2} \sin ^{2} \vartheta
$$

Harding \& Daugherty (1991) already discussed the solutions to this equation, which have to be found numerically and are often being referred to as zero line width solutions. They are shown

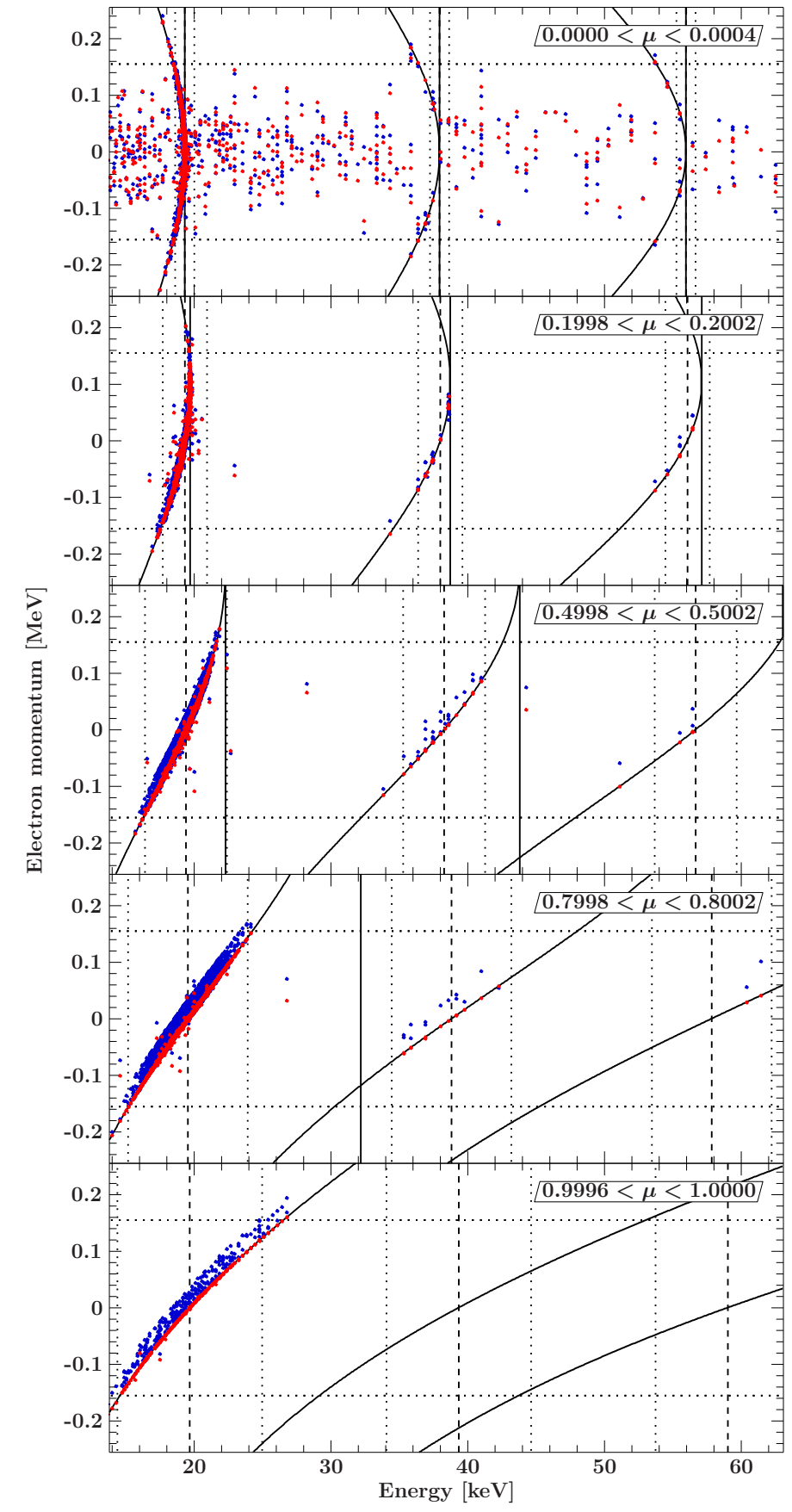

Fig. 5. Sampled electron parallel momenta for one MC run. Each point represents one scattered photon by its energy on the $x$-axis and the corresponding electron's momentum before (red) and after (blue) the scattering process on the $y$-axis. Scattering events involving photons incoming at angles of $\sim 90^{\circ}, 79^{\circ}, 60^{\circ}, 37^{\circ}$, and $1^{\circ}$ to the magnetic field (from top to bottom) are shown. The setup of the calculation is the same as in Isenberg et al. (1998) with a magnetic field $B=0.0385 B_{\text {crit }}$, electron parallel temperature $k_{\mathrm{B}} T=5 \mathrm{keV}$, and a bottom-illuminated slab geometry of the line-forming region. Vertical dashed lines show the cyclotron line energy, which is slightly shifted to higher energies for smaller angles to the magnetic field, because of the angular dependence of the resonance energy. This shift is often neglected by observers in favor of using the simplified $12-B-12$ rule. Solid vertical lines mark the position of the cut-off energy beyond which no resonant scattering is possible. Dotted horizontal lines enclose the electron momentum range in which 99\% of the electrons are located (assuming a relativistic Maxwellian, Eq. (3)). Solid curved lines show the possible solutions for resonant scattering. The vertical dotted lines indicate the Doppler width of the cyclotron lines (see Eq. (9)). 
as curved lines in Fig. 5. The resonance condition can only be fulfilled for photon energies below the cut-off energy,

$\omega_{\text {cut }}(n)=\frac{\sqrt{1+2 n B / B_{\text {crit }}}-1}{\sin \vartheta} m_{\mathrm{e}} c^{2}$.

The vertical solid lines in Fig. 5 mark the corresponding positions of the cut-off energies. No interaction involving the excitation of an electron to Landau level $n$ occurs beyond that energy. In the following we describe the zero line width solutions in energy-momentum space and provide physically motivated regimes in which these solutions are expected to be fulfilled, that is, energy and momentum limits. We show, for the first time, simulated MC scattering events in the context of these zero line width solutions (and their limits) with the aim to establish an understanding of the complex cyclotron scattering process. Furthermore, we use this representation as a consistency check for our MC simulation. Schwarm (2010) performed a similar check using the MC code by Araya (1997) without the physically motivated limits in energy and momentum space. Only photons moving upwards or almost perpendicular to the column axis are shown, which is why no decrease in electron momentum occurs. Numerically calculated solutions of Eq. (7) are shown in Fig. 5 as black solid curves. Vertical solid lines mark the positions of the cut-off energies corresponding to the three cyclotron resonances shown. Positive and negative electron momenta are possible to fulfill the resonance condition for photons with incoming angles almost perpendicular to the magnetic field and energies below the cut-off energy. At the cut-off energy a unique solution is possible for electrons with zero parallel momentum. For smaller angles to the magnetic field the momentum solutions deform, following the cut-off energy which is shifting to higher energies. The sampled events are bound to a limited region of the energy-momentum space. The cyclotron resonance widths provide boundaries on the energy axes, since only photons whose momentum averaged mean free path is small enough for scattering to take place will appear in the figure. As an estimate, we use the full Doppler width for thermal cyclotron line broadening (Meszaros \& Nagel 1985),

$\frac{\Delta E}{E}=\sqrt{8 \ln 2 \frac{k_{\mathrm{B}} T}{m_{\mathrm{e}} c^{2}}} \cos \vartheta$

The electron momentum space is limited by the width of the relativistic Maxwellian momentum distribution. We use its definition in Eq. (3) to calculate the momentum range in which $99 \%$ of the initial electrons reside. Therefore, the plasma temperature is the main parameter responsible for a limited spreading of scattering events in energy-momentum space (Mészáros 1978). The boundaries of the energy-momentum space available for scattering events are shown in Fig. 5 as dotted vertical and horizontal lines respectively.

Finally, the dashed lines in Fig. 5 show the cyclotron line energies, that is, where $n(\omega, \mu)$ is an integer (see Eq. (A.3)). For photons moving perpendicular to the $B$-field this line overlaps with the cut-off energy.

Comparing the red points in Fig. 5, which mark the initial parallel momentum, with the blue points, which correspond to the electron momentum after the scattering process, shows that the electrons gain parallel momentum in the (projected) direction of the incoming photon. For angles perpendicular to the magnetic field there is no continuous momentum transfer, while for photons incoming at smaller angles to the magnetic field parallel momentum is transferred. Figure 5 shows only photons moving upwards. The electron momenta for scattering processes with a parallel component are therefore shifted to higher positive momenta.

The number of scattering events declines for very small photon angles to the magnetic field, from $\sim 4000$ scattering events in the top panel to $\sim 300$ events in the bottom panel. The reason for this is twofold: first, the almost zero photon momentum component perpendicular to the field leads to a decrease of resonant cyclotron scattering events and second, repeated cyclotron scattering favors a redistribution of the interacting photons towards larger angles to the $B$-field, especially for photon energies close to the cyclotron energy. A detailed description of the classic CRSF geometries will be provided in Paper II.

Now we are able to verify that scattering events are of resonant nature (i.e., they involve an excitation of an electron), the resonant scattering events coincide with the zero line width solutions, and the final electron momentum is increasing with decreasing scattering angle. The points which are not located on the zero line width solutions mark elastic scattering processes not involving any electron excitations. They occur mainly close to $\vartheta \sim 90^{\circ}$ where resonant scattering is suppressed because the photons have to have exactly the right energy to excite an electron. Photons with a smaller angle to the magnetic field, on the other hand, can transfer excessive energy to the electron's momentum parallel to the field. Therefore the red and blue points of the resonant scattering events are almost congruent in the top panel of Fig. 5, while the gap between them increases with increasing $\mu=\cos \vartheta$. The data show that our physically motivated limits provide accurate boundaries for the majority of scattering events and further justifies the usage of Eq. (9) as an approximation to the CRSF line width.

\section{Summary}

For accelerating Monte Carlo simulations of cyclotron lines we replaced the most time consuming part - that is the calculation of the photon mean free path - with a tabular interpolation scheme. These tables store the mean free paths of photons for different incident angles and energies. The partial results necessary for interpolating the electron momentum after a mean free path has been drawn from the exponential probability distribution, and the spin dependent results, are saved as well. The electronic tables described here are available online (see link in footnote, page 1).

This interpolation scheme is used to generate synthetic cyclotron line spectra using our MC simulation code. It enables us to simulate much more complex physical scenarios than previous works. As an example, we have investigated the application of the momentum sampling.

Acknowledgements. This work has been partially funded by the Deutsche Forschungsgemeinschaft under DFG grant number WI 1860/11-1 and by the Deutsches Zentrum für Luft- und Raumfahrt under DLR grant numbers 50 OR 1113, 50 OR 1207, and 50 OR 1411. M.T.W. is supported by the Chief of Naval Research and by the National Aeronautics and Space Administration Astrophysical Data Analysis Program. We thank the International Space Science Institute in Bern for inspiring team meetings. The fruitful discussions within the MAGNET collaboration also had a very positive impact on this work.

\section{References}

Araya, R. A. 1997, Ph.D. Thesis, Johns Hopkins University, Baltimore Araya, R. A., \& Harding, A. K. 1999, ApJ, 517, 334 Becker, P. A., \& Wolff, M. T. 2007, ApJ, 654, 435 Bonazzola, S., Heyvaerts, J., \& Puget, J. L. 1979, A\&A, 78, 53 
F.-W. Schwarm et al.: Cyclotron resonant scattering feature simulations. I.

Bussard, R. W., Alexander, S. B., \& Meszaros, P. 1986, Phys. Rev. D, 34, 440 Canuto, V., Lodenquai, J., \& Ruderman, M. 1971, Phys. Rev. D, 3, 2303

Daugherty, J. K., \& Harding, A. K. 1986, ApJ, 309, 362

Daugherty, J. K., \& Ventura, J. 1978, Phys. Rev. D, 18, 1053

Gonthier, P. L., Baring, M. G., Eiles, M. T., et al. 2014, Phys. Rev. D, 90, 043014

Graziani, C. 1993, ApJ, 412, 351

Harding, A. K., \& Daugherty, J. K. 1991, ApJ, 374, 687

Herold, H., Ruder, H., \& Wunner, G. 1982, A\&A, 115, 90

Isenberg, M., Lamb, D. Q., \& Wang, J. C. L. 1998, ApJ, 505, 688

Johnson, M. H., \& Lippmann, B. A. 1949, Phys. Rev., 76, 828

Langer, S. H. 1981, Phys. Rev. D, 23, 328

Latal, H. G. 1986, ApJ, 309, 372

Lyness, J. N. 1969, J. Comm. Assoc. Computing Machinery, 16, 483
McKeeman, W. M. 1962, Comm. Assoc. Computing Machinery, 5, 604 Mészáros, P. 1978, A\&A, 63, L19

Meszaros, P., \& Nagel, W. 1985, ApJ, 298, 147

Mészáros, P., \& Ventura, J. 1978, Phys. Rev. Lett., 41

MPI Forum 1994, MPI: A Message-Passing Interface Standard, Technical Report FUT-CS-94-230, University of Tennessee, Knoxville, TN

Schönherr, G., Wilms, J., Kretschmar, P., et al. 2007, A\&A, 472, 353

Schwarm, F.-W. 2010, Diploma Thesis, Univ. Erlangen-Nuremberg

Schwarm, F.-W., Schönherr, G., Wilms, J., \& Kretschmar, P. 2012, PoS, INTEGRAL 2012, 153

Sina, R. 1996, Ph.D. Thesis, University of Maryland, College Park, MD

Sokolov, A. A., Ternov, I. M., Bagrov, V. G., Gal'tsov, D. V., \& Zhukovskii, V. C. 1968, Sov. Phys., 11, 4 


\section{Appendix A: Interpolation techniques}

In the following we explain the interpolation techniques used for the creation of the MFP tables and to interpolate from them during CRSF simulations with our new MC code.

As discussed above, the interpolation tables were refined adaptively by comparing the calculated profile $\sigma_{\text {calc }}$ of a new point with the profile obtained by interpolation from the table $\sigma_{\text {interp }}$, without taking this newly calculated point into account. A linear interpolation scheme was used for refining the energy grid, that is

$\langle\sigma\rangle_{\text {interp }}(\mu, \omega)=\langle\sigma\rangle\left(\mu, \omega_{1}\right)+\frac{\langle\sigma\rangle\left(\mu, \omega_{2}\right)-\langle\sigma\rangle\left(\mu, \omega_{1}\right)}{\omega_{2}-\omega_{1}}\left(\omega-\omega_{1}\right)$.

The splitting of the energy intervals is stopped if

$\frac{\langle\sigma\rangle_{\text {interp }}-\langle\sigma\rangle_{\text {calc }}}{\langle\sigma\rangle_{\text {calc }}} \leq \epsilon$,

where $\epsilon=1 / 15$ has been found to be a good compromise between precision and calculation time.

The same linear interpolation method can be used to interpolate profiles during Monte Carlo simulations. For an interpolation in $\mu$ and $\omega$ two linear interpolations have to be performed. First the inverse mean free path is interpolated for the surrounding angular grid points, each one on its own energy grid. Linear interpolation between these values with respect to the angle then gives the desired value.

For using the computed mean free path tables for the simulation of synthetic spectra with CRSFs, a physically motivated interpolation method was implemented as well. Instead of interpolating for the same energy on two different angular grid points, the energy is shifted to the corresponding energy of the angular point considered. This is done by calculating the order $n$ of the energy in terms of the resonance condition for the desired angular value. This value is used to calculate the corresponding energies at the angular boundaries used for interpolation.

$n(\omega, \mu)=\frac{\left(\omega \sin ^{2} \vartheta / m_{\mathrm{e}} c^{2}+1\right)^{2}-1}{2 B / B_{\text {crit }} \sin ^{2} \vartheta}$.

This interpolation scheme eliminates inaccuracies due to the shift of the resonance energy with $\vartheta$, because interpolation is done at nearly constant "elevation" along the resonance ridges (Harding \& Daugherty 1991).

\section{Appendix B: Mean free path table structure}

The naming convention of the mean free path tables was chosen as follows: all names start with $\mathrm{mfp}_{-}$followed by the table specific parameters, namely the magnetic field strength $B$ and the temperature $T$. The magnetic field is in units of the critical magnetic field strength, with five digits The temperature $k_{\mathrm{B}} T$ is given in units of $\mathrm{MeV}$ with five digits as well.
Table B.1. List of FITS keywords.

\begin{tabular}{cl}
\hline \hline Name & Description \\
\hline $\mathrm{B}$ & Magnetic field $\left[B_{\text {crit }}\right]$ \\
$\mathrm{T}$ & Electron temperature $k_{\mathrm{B}} T[\mathrm{MeV}]$ \\
$\mathrm{MU}$ & $\begin{array}{l}\text { Cosine of the incoming photons angle to the } \\
\text { magnetic field, } \mu=\cos \vartheta\end{array}$ \\
MAX_ERR & Maximum relative error in units of $1 / 15$ \\
\hline
\end{tabular}

Table B.2. Uncompressed file sizes for the parameter combinations made available in GB.

\begin{tabular}{crcccc}
\hline \hline & \multicolumn{5}{c}{$B / B_{\text {crit }}$} \\
$k_{\mathrm{B}} T[\mathrm{keV}]$ & 0.01 & 0.03 & 0.06 & 0.09 & 0.12 \\
\hline 3 & 44 & 16 & 10 & 7 & 11 \\
6 & 89 & 29 & 16 & 11 & 16 \\
9 & 148 & 42 & 23 & 16 & 22 \\
12 & 198 & 61 & 32 & 20 & 26 \\
15 & 238 & 74 & 35 & 24 & 32 \\
\hline
\end{tabular}

Notes. Compression reduces the file size by approximately $50 \%$.

For example, the first table in alphabetical order is named mfp_BO.0100TO.0030.fits.

Each mean free path table starts with an empty image HDU containing header keywords for the magnetic field strength $B$, the temperature $T$, and the relative maximum error as listed in Table B.1. It is followed by a variable number of binary extensions any of which corresponds to one angular grid point. These binary extensions are ordered by increasing $\mu=\cos \vartheta$ and are described in detail in the following.

The value of $\mu$ is stored in the header keyword MU. Each row corresponds to one energy grid point, the value of which can be found in the first column. The second column contains the total thermally averaged scattering cross section summed over final electron spin states. The number of grid points used for the adaptive Simpson integration over the thermal electron momentum distribution (Eq. (2)), can be found in the third column. The corresponding electron momentum grid points are stored in the fourth column as a variable length array of double numbers, followed by the spin averaged cumulative distribution function (CDF), the ensemble of partial integrals obtained from Eq. (5). The sixth column contains, again, the number of electron momentum grid points used for the integration of Eq. (2) taking into account only transitions with final electron spin down. Following the corresponding momentum grid points and cumulative distribution functions the pattern repeats once again for the case of final electron spin up.

The header keyword MAX_ERR contains the relative error of the table, that is, $\epsilon$ from Eq. (A.2), in units of $1 / 15$.

Table B. 3 visualizes the structure described here. The last six columns for the spin dependent cases are omitted for the sake of clarity. 
F.-W. Schwarm et al.: Cyclotron resonant scattering feature simulations. I.

Table B.3. Description of the mean free path table structure for given values of $k_{\mathrm{B}} T$ and $B / B_{\text {crit }}$.

\begin{tabular}{ccccccc}
\hline \hline EXTENSION & MU & \multicolumn{5}{c}{ COLUMNS } \\
\hline \multicolumn{1}{c}{ Energy $[\mathrm{MeV}]$} & Cross section $\left[\sigma_{\mathrm{Th}}\right]$ & \# of grid points & Grid $[\mathrm{MeV}]$ & CDF \\
\hline 1 & $\mu_{1}$ & $k_{1}$ & $\left\langle\sigma\left(\mu_{1}, k_{1}\right)\right\rangle$ & $N_{p}$ & {$\left[p_{1}, \ldots, p_{N_{p}}\right]$} & {$\left[F_{-m_{\mathrm{e}} c}^{p_{1}}, \ldots, F_{-m_{\mathrm{e}} c}^{p_{N_{p}}}\right]$} \\
& & $\vdots$ & & & & \\
& & $k_{N_{k}}$ & $\left\langle\sigma\left(\mu_{1}, k_{N_{k}}\right)\right\rangle$ & $N_{p}$ & {$\left[p_{1}, \ldots, p_{N_{p}}\right]$} & {$\left[F_{-m_{\mathrm{e}} c}^{p_{1}}, \ldots, F_{-m_{\mathrm{e}} c}^{p_{N_{p}}}\right]$} \\
$\vdots$ & $\vdots$ & $: !$ & $: \vdots$ & $:$ & $:$ \\
$N_{\mu}$ & $\mu_{N_{\mu}}$ & $k_{1}$ & $\left\langle\sigma\left(\mu_{N_{\mu}}, k_{1}\right)\right\rangle$ & $N_{p}$ & {$\left[p_{1}, \ldots, p_{N_{p}}\right]$} & {$\left[F_{-m_{\mathrm{e}} c}^{p_{1}}, \ldots, F_{-m_{\mathrm{e}} c}^{p_{N_{p}}}\right]$} \\
& & $\vdots$ & & & & \\
& & $k_{N_{k}}$ & $\left\langle\sigma\left(\mu_{N_{\mu}}, k_{N_{k}}\right)\right\rangle$ & $N_{p}$ & {$\left[p_{1}, \ldots, p_{N_{p}}\right]$} & {$\left[F_{-m_{\mathrm{e}} c}^{p_{1}}, \ldots, F_{-m_{\mathrm{e}} c}^{p_{N_{p}}}\right]$} \\
\hline
\end{tabular}

Notes. The column pattern \# of grid points, Grid [MeV], CDF is repeated two more times, omitted below for clarity, for the case of final electron spin down and final electron spin up, respectively. The first element of each Grid and CDF array is used for internal consistency checks. The user should only use the indices 1 to $N_{p}$. The dependency of $N_{p}$ on angle and energy is not stated explicitly. $N_{\mu}, N_{k}$, and $N_{p}$ are typically on the order of a few hundred to thousand of points.

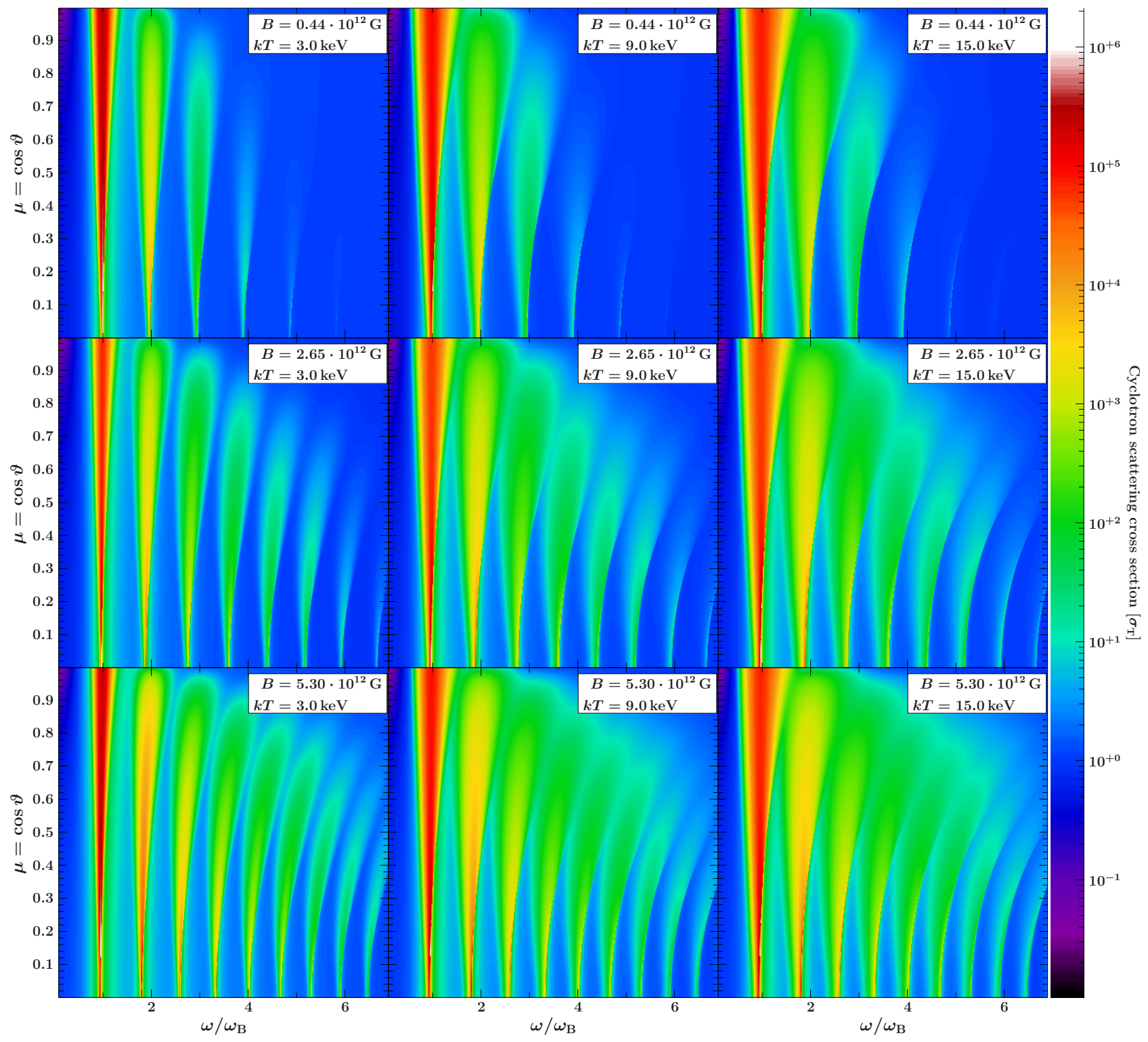

Fig. B.1. Visualization of the adaptively calculated inverse mean free path values from the interpolation tables for $B=0.01,0.06$, and $0.12 B_{\text {crit }}$. The colors correspond to the averaged scattering cross sections. 


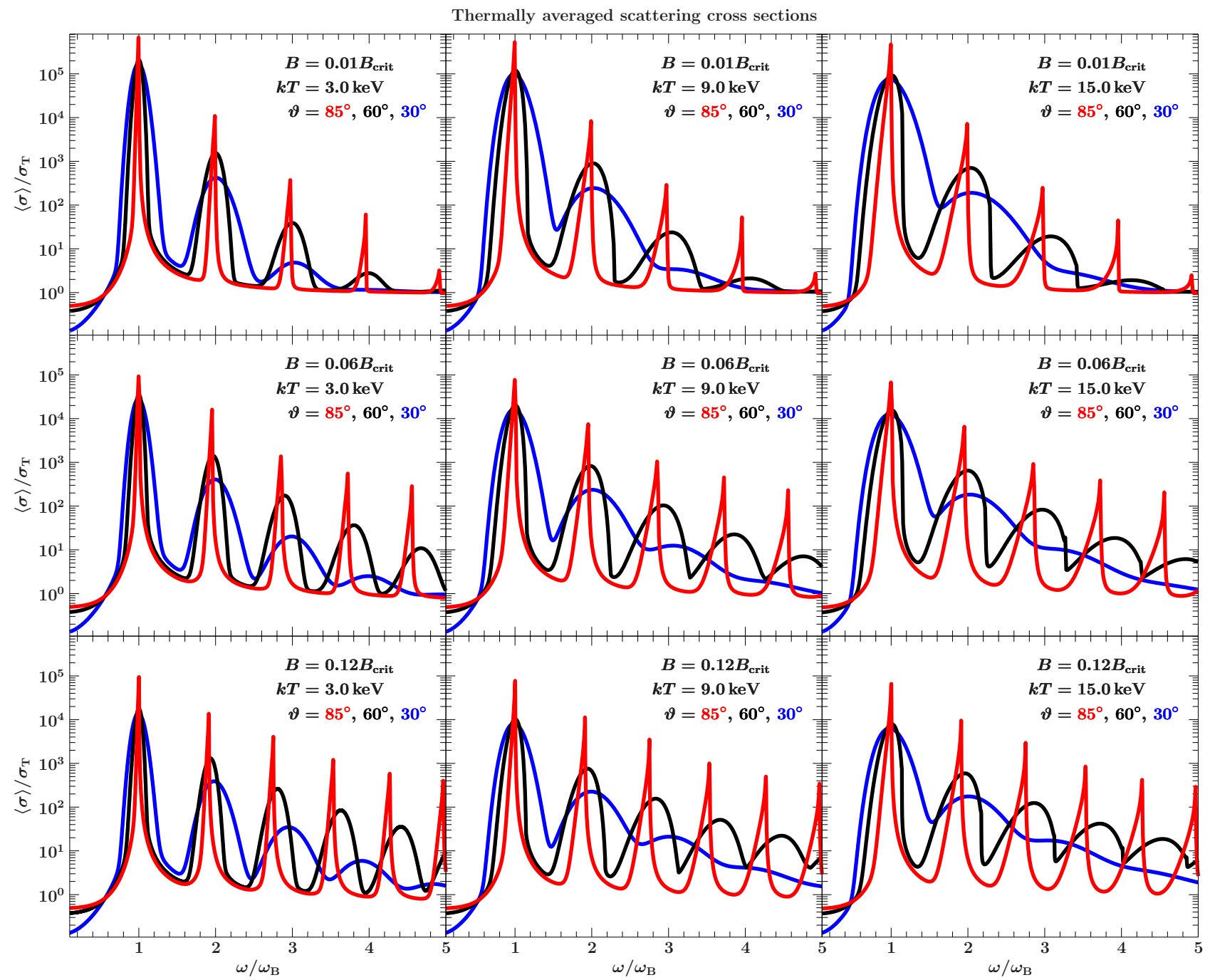

Fig. B.2. Adaptively calculated averaged cyclotron resonance scattering cross sections. The colors correspond to different angles of the scattering photon with respect to the magnetic field. 